\title{
NEW X-RAY-SELECTED PRE-MAIN-SEQUENCE MEMBERS OF THE SERPENS MOLECULAR CLOUD
}

\author{
Isa Oliveira ${ }^{1,4}$, Margriet VAN DER LaAn ${ }^{2}$, AND JoAnNa M. Brown ${ }^{3}$ \\ ${ }^{1}$ McDonald Observatory, The University of Texas at Austin, 1 University Station, C1402, Austin, TX 78712, USA; oliveira@ astro.as.utexas.edu \\ ${ }^{2}$ Leiden Observatory, Leiden University, P.O. Box 9513, 2300 RA Leiden, Netherlands \\ ${ }^{3}$ Harvard-Smithsonian Center for Astrophysics, 60 Garden Street, MS 78, Cambridge, MA 02138, USA \\ Received 2013 May 16; accepted 2013 August 8; published 2013 September 20
}

\begin{abstract}
The study of young stars no longer surrounded by disks can greatly add to our understanding of how protoplanetary disks evolve and planets form. We have used VLT/FLAMES optical spectroscopy to confirm the youth and membership of 19 new young diskless stars in the Serpens Molecular Cloud, identified at X-ray wavelengths. Spectral types, effective temperatures, and stellar luminosities were determined using optical spectra and optical/near-infrared photometry. Stellar masses and ages were derived based on pre-main-sequence evolutionary tracks. The results yield remarkable similarities for age and mass distribution between the diskless and disk-bearing stellar populations in Serpens. We discuss the important implications these similarities may have on the standard picture of disk evolution.
\end{abstract}

Key words: circumstellar matter - Hertzsprung-Russell and C-M diagrams - ISM: individual objects (Serpens) - stars: pre-main-sequence

Online-only material: color figures

\section{INTRODUCTION}

The manner in which protoplanetary disks evolve and, potentially, form planets is one of the most exciting subjects of research in astrophysics in the last decade. Through multiwavelength observations, combined with a growing number of models and theories, many complex processes have been found to play a role in explaining how planets could possibly form from the small-sized dust and gas initially present in those disks. It is not yet clear exactly under which circumstances the different processes are determinant. However, it is clear that the study of protoplanetary disks alone is unlikely to lead to a full picture of disk evolution.

A powerful way to learn more about disks is to study young stars that are no longer surrounded by them. It is believed that all stars are born harboring circumstellar disks, through conservation of angular momentum during the star formation process. Observations have shown that not all disks have the same lifetime; they vary between approximately 1 and $10 \mathrm{Myr}$, with about $50 \%$ of dusty disks disappearing within $3 \mathrm{Myr}$ (e.g., Haisch et al. 2001; Hernández et al. 2008). Differences between young stars of the same age with and without disks could yield a greater understanding of the processes that affect disk evolution and eventually allow time for planets to form.

The difficulty in studying young stars without disks lies in identifying them. Young stars are most commonly found by their colors, which takes into account the presence of the dusty disk emission, prominent in the infrared (IR) region of the system (star+disk) spectrum. If a young star is no longer surrounded by a disk, its optical-IR colors are indistinguishable from foreground/background stars observed in the same direction as the star-forming region to which it belongs. With broadband optical/IR photometric studies, it can be difficult to identify candidate diskless young stars.

The picture changes considerably when studying a region at X-ray wavelengths. In this short-wavelength regime, young

\footnotetext{
4 Harlan J. Smith Postdoctoral Fellow.
}

stars are very active whether or not a disk is still present (e.g., Preibisch et al. 2005; Feigelson et al. 2007). Indeed, X-ray emission has often been used to identify young stellar objects in the literature (e.g., Feigelson \& Kriss 1981; Walter \& Kuhi 1981; Feigelson \& Montmerle 1999; Getman et al. 2005; Padgett et al. 2006; Güdel et al. 2007; Winston et al. 2007; Telleschi et al. 2007). Confirmation of their young stellar nature, however, is necessary through other means, the most powerful of which being optical spectroscopy (e.g., Walter et al. 1988; Scelsi et al. 2008).

The Serpens Molecular Cloud is a young star-forming region ( $\sim 2 \mathrm{Myr}$; Oliveira et al. 2013). Very long baseline interferometry measurements by Dzib et al. (2010) of EC95 in the Serpens Core indicate it at a distance of $415 \mathrm{pc}$. Given the size of the cloud, it is reasonable to assume a distance of $415 \pm 15 \mathrm{pc}$ for its entirety. The young stellar population still surrounded by disks in Serpens beyond its core has been uncovered in the IR by the Spitzer Space Telescope Legacy Program "c2d" (Harvey et al. 2006, 2007a, 2007b). Unlike that in the Serpens Core, which has been well studied in a variety of wavelengths (e.g., Winston et al. 2007, 2009, 2010), nothing was known about the diskless young stellar population of Serpens beyond its core until it was observed with XMM-Newton (J. M. Brown et al., in preparation). Two XMM-Newton pointings cover about twothirds of the Serpens area observed by the c2d, and dozens of objects were identified by Brown et al. as young diskless star candidates, for which confirmation is still necessary.

In this paper we report on an optical multi-object spectroscopic survey designed to confirm the youth and membership of those newly discovered young diskless star candidates discovered in Serpens with XMM-Newton. Section 2 describes the VLT/FLAMES observations and data reduction. The data are analyzed in Section 3, where spectral types, stellar temperatures, and luminosities are determined. Spectral energy distributions (SEDs) are constructed with the addition of optical and nearand mid-IR photometry from the literature. Then, the objects are placed in an H-R diagram overlaid with pre-main-sequence (PMS) evolutionary tracks, from which individual ages and 
Table 1

Observational Log

\begin{tabular}{lclc}
\hline \hline Obs. Block ID & Field & Obs. Night & $\begin{array}{c}\text { Exp. Time } \\
(\mathrm{s})\end{array}$ \\
\hline 357791 & Serpens AO5 & 2009 Jun 11 & 2000 \\
357791 & Serpens AO5 & 2009 Jun 11 & 2000 \\
357792 & Serpens AO5 & 2009 Jun 11 & 2000 \\
357792 & Serpens AO5 & 2009 Jun 11 & 2000 \\
357792 & Serpens AO5 & 2009 Jun 11 & 2000 \\
357793 & Serpens AO6 & 2009 Jun 8 & 2000 \\
357793 & Serpens AO6 & 2009 Jun 8 & 2000 \\
365063 & Serpens AO6 & 2009 Jun 11 & 2000 \\
365063 & Serpens AO6 & 2009 Jun 11 & 2000 \\
\hline
\end{tabular}

masses are derived. In Section 4, the bona-fide diskless young stellar population of Serpens is discussed and contextualized with respect to the disk-bearing population (Oliveira et al. 2009, 2010, 2013). Finally, we present our conclusions in Section 5.

\section{OBSERVATIONS AND DATA REDUCTION}

The spectroscopic data were obtained using FLAMES on the 8.2 m UT2 (Kueyen) telescope of ESO, in Paranal, Chile, in service mode in 2009 June. FLAMES consists of three components: a fiber positioner (OzPoz), a medium- to highresolution optical spectrograph GIRAFFE, and a link to the instrument UVES. The data used in this work consist of two observing blocks from FLAMES/GIRAFFE using the MEDUSA fibers. This setting allows for up to 132 fibers of $1^{\prime \prime} .2$ aperture in the $25^{\prime}$ diameter field of view of the instrument. The wavelength range chosen, 6437-7183 , offers a spectral resolution of $0.8 \AA$ and covers temperature-sensitive features that allow spectral classification.

The two observed fields (AO5 and AO6) were centered on the $X M M$-Newton observations. An input list of the young stellar candidates' coordinates was provided based on X-ray detections from J. M. Brown et al. (in preparation). AO5, observed by XMM-Newton on 2007 April 15 in program 0402820101, was centered at $277.254625+0.49727778$, and AO6, observed on 2008 April 16 in XMM program 0503240201, was centered at $277.34775+0.8228333$. XMM-Newton has a slightly larger aperture than FLAMES, $30^{\prime}$ versus $25^{\prime}$, so candidates on the edges of the XMM fields could not be observed. All X-ray sources, regardless of optical or IR characteristics, were considered for follow up. Preference was given to targets with optical counterparts that are not part of the Spitzer disk sample in the same region (Harvey et al. 2006, 2007a, 2007b) and had no previously known spectral information. Fiber placement was optimized using the FPOSS fiber configuration program of FLAMES, which permitted observation of most candidates. However, some candidates could not be simultaneously observed and had to be dropped. Unused fibers were placed on "blank" positions of the sky for sky subtraction. To maximize dynamic range, fields were observed using multiple 2000 s exposures. Field AO5 had five such exposures and field AO6 had four. A summary of the observational details can be found in Table 1 .

Data reduction was performed within the ESO reduction pipeline GASGANO. The pipeline performs frame correction for detector defects, fiber localization and tracing, flat-fielding and fiber transmission, correction of scattered light, and wavelength calibration. The science spectra were extracted within IDL after wavelength calibration. For each observed field, all sky spectra were combined with a $2 \sigma$ clipping in order to exclude cosmic rays or any other artifacts. This combined sky spectrum was then subtracted from the science spectra to remove sky lines. Since each field was observed more than once (see Table 1), all observed spectra of the same object are combined, also with a $2 \sigma$ clipping, leading to the final spectrum of each object. Figure 1 shows a representative sample of the spectra of the objects in this sample.

The final data set consists of 86 observed objects, 26 from field AO5 and 60 from field AO6 (details in Tables 2 and 3, respectively). However, 61 of these objects turned out to be much too faint and their spectra are underexposed, without enough signal-to-noise ratio $(\mathrm{S} / \mathrm{N})$ to show any of the features necessary for spectral classification $(\mathrm{S} / \mathrm{N} \gtrsim 10$ per pixel). These objects are marked as low $\mathrm{S} / \mathrm{N}$ in Tables 2 and 3 and are left out of further analysis.

\section{DATA ANALYSIS}

The positions of the 25 objects analyzed are shown in Figure 2. The 7 objects in field AO5 are shown in blue, while the 18 objects in AO6 are in red. The Serpens members with disks, as observed with Spitzer by Oliveira et al. (2010), are shown in gray for comparison.

It is worth noting that the spectra presented here cover the $\mathrm{H} \alpha$ line, an indicator of mass accretion onto the star (White \& Basri 2003; Muzerolle et al. 2003; Natta et al. 2004), and the Li I absorption line, often used as a general indicator of stellar youth. Lithium can be depleted in low-mass stars (late-K and $M$ types) due to fusion in the stellar interior. Evolutionary models predict depletion to start at $\sim 10-20 \mathrm{Myr}$ and proceed quickly. Stars more massive than $\sim 1 M_{\odot}$ develop radiative cores, limiting mixing to the center, which retards depletion (Randich et al. 2001; Hartmann 2003). Due to variations in S/N, Li I could not be detected in all of the spectra presented here. The detection criterion adopted is an equivalent width greater than $0.1 \AA$. The presence (in emission or absorption) or absence of these lines, as well as the presence of IR excess in those sources, are indicated in Table 4.

\subsection{Spectral Types}

Spectral classification of the 25 objects is performed by comparing each spectrum to a library of standards, following Oliveira et al. (2009) and Mortier et al. (2011). The routine used here classifies the optical spectra by a direct comparison with the grid of standards from EXPORT (Mora et al. 2001) and one from Montes et al. (1997). In this routine the science spectra are first normalized to the continuum and then overplotted on the normalized standards of different spectral types. A $\chi^{2}$ minimization is performed to find the best fit among all spectral types, followed by a visual inspection of each object. This method yields an accuracy of a half spectral class given the range of standard spectra available. A larger uncertainty is found for the spectra with lower $\mathrm{S} / \mathrm{N}$. The distribution of spectral types from this work is shown in Figure 3, while the results for individual stars can be found in Table 4.

Similar to the distribution of spectral types of the young stars with disks (Oliveira et al. 2009), the majority of stars in this sample are of late $\mathrm{K}$ and $\mathrm{M}$ spectral types, with only a few being earlier G-types.

\subsection{Stellar Temperatures}

Effective temperatures ( $T_{\text {eff }}$ ) for the individual objects were determined using calibrations that relate them to spectral types. For stars of a type earlier than M0, the relationship established 

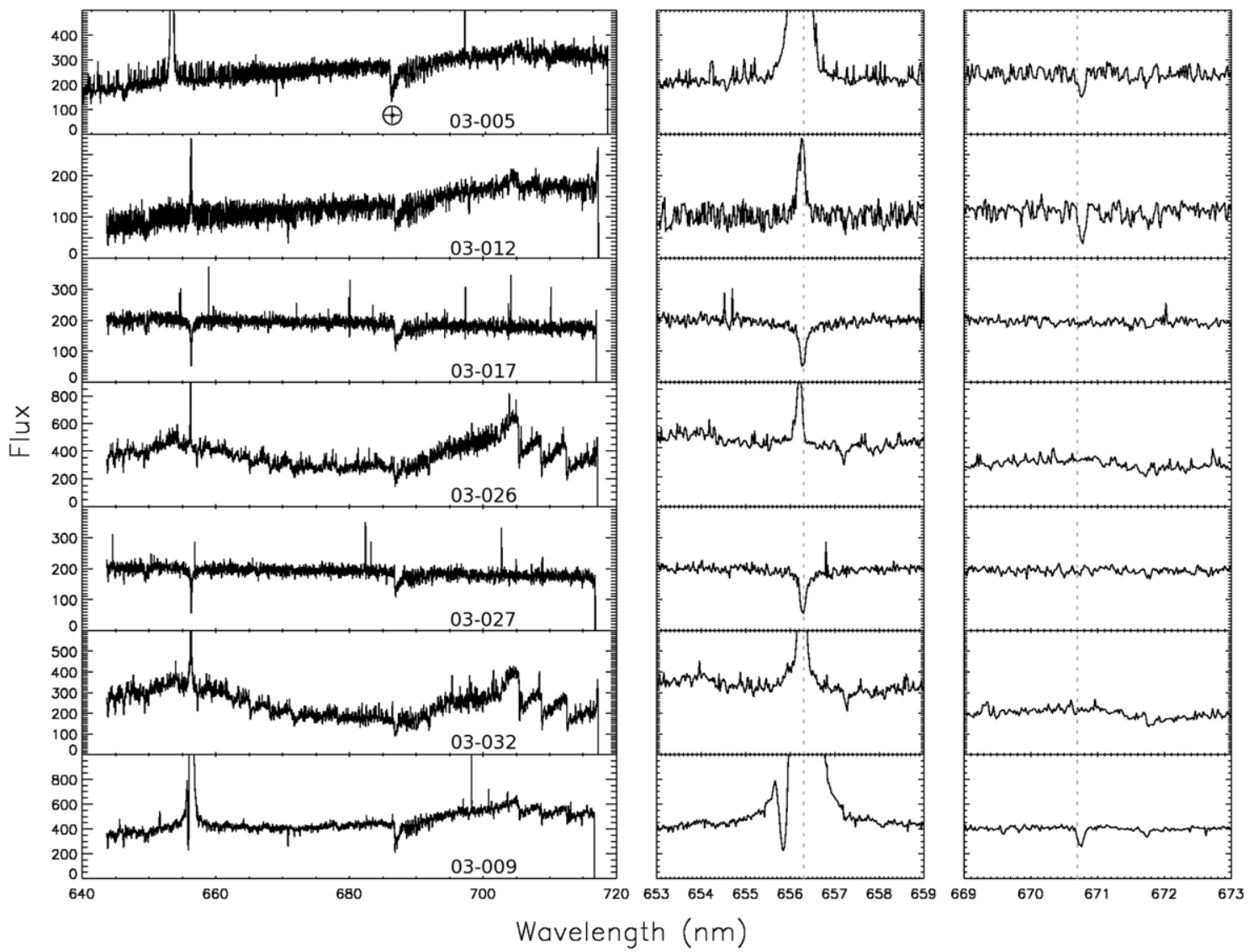

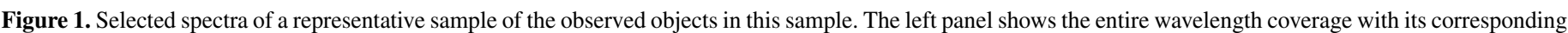

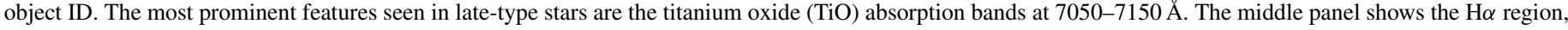
and the right panel shows the Li I line.

Table 2

Observed Objects in Field AO5

\begin{tabular}{|c|c|c|c|c|c|c|c|c|c|}
\hline $\mathrm{ID}^{\mathrm{a}}$ & $\begin{array}{l}\text { R.A. } \\
\text { (deg) }\end{array}$ & $\begin{array}{l}\text { Decl. } \\
\text { (deg) }\end{array}$ & 2MASS Name & $\begin{array}{c}R \\
(\mathrm{mag})\end{array}$ & $\begin{array}{c}Z \\
(\mathrm{mag})\end{array}$ & $\begin{array}{c}J \\
(\mathrm{mag})\end{array}$ & $\begin{array}{c}H \\
(\mathrm{mag})\end{array}$ & $\begin{array}{c}K \\
(\mathrm{mag})\end{array}$ & Good S/N? \\
\hline 03-005 & 277.39835 & 0.584499 & $18293563+0035035$ & 18.2 & 14.3 & 12.0 & 10.8 & 10.3 & Yes \\
\hline 03-007 & 277.29083 & 0.492694 & $18290985+0029329$ & 19.2 & 15.6 & 12.4 & 10.8 & 10.1 & No \\
\hline 03-008 & 277.24176 & 0.290611 & $18285808+0017243$ & 21.9 & 18.0 & 10.7 & 9.8 & 9.4 & No \\
\hline 03-009 & 277.25363 & 0.492055 & $18290088+0029312$ & 17.0 & 14.1 & 11.6 & 10.0 & 9.0 & Yes \\
\hline 03-011 & 277.37317 & 0.558027 & $18292959+0033280$ & 20.8 & 16.0 & 13.7 & 12.6 & 12.1 & No \\
\hline 03-012 & 277.37091 & 0.302722 & $18292905+0018091$ & 17.2 & 14.7 & 12.4 & 11.4 & 10.9 & Yes \\
\hline 03-013 & 277.28870 & 0.304611 & $18290934+0018159$ & 22.4 & 18.3 & 14.4 & 12.0 & 11.0 & No \\
\hline 03-014 & 277.27942 & 0.643972 & $18290698+0038378$ & 21.9 & 18.0 & 15.2 & 12.9 & 11.5 & No \\
\hline 03-015 & 277.43549 & 0.565333 & $18294460+0033553$ & 19.4 & 15.7 & 12.7 & 11.9 & 11.5 & No \\
\hline 03-017 & 277.34201 & 0.672361 & $18292209+0040203$ & 23.0 & 17.4 & 13.3 & 11.1 & 10.0 & Yes \\
\hline 03-020 & 277.26788 & 0.556611 & $18290437+0033235$ & 21.9 & 18.0 & 15.9 & 13.0 & 11.3 & No \\
\hline 03-021 & 277.35974 & 0.501416 & $18292641+0030043$ & 21.9 & 18.0 & 16.2 & 13.9 & 12.9 & No \\
\hline $03-022$ & 277.25016 & 0.282972 & $18290025+0016578$ & 17.7 & 15.2 & 12.9 & 11.5 & 10.7 & No \\
\hline 03-023 & 277.32410 & 0.297277 & $18291770+0017488$ & 21.9 & 18.0 & 13.3 & 12.7 & 12.4 & No \\
\hline 03-025 & 277.40137 & 0.565749 & $18293634+0033559$ & 21.4 & 16.3 & 13.8 & 12.3 & 11.6 & No \\
\hline 03-026 & 277.28122 & 0.456777 & $18290748+0027234$ & 17.0 & 14.7 & 13.2 & 12.6 & 12.3 & Yes \\
\hline 03-027 & 277.31396 & 0.658166 & $18291521+0039343$ & 20.4 & 16.1 & 13.4 & 12.1 & 11.5 & Yes \\
\hline 03-028 & 277.17624 & 0.627361 & $18283136+0030534$ & 21.9 & 18.0 & 14.4 & 13.0 & 12.3 & No \\
\hline 03-029 & 277.27597 & 0.329388 & $18290615+0019442$ & 18.8 & 15.6 & 13.4 & 12.2 & 11.8 & No \\
\hline 03-030 & 277.43771 & 0.540333 & $18294516+0032245$ & 20.3 & 16.6 & 13.2 & 11.9 & 11.3 & No \\
\hline 03-032 & 277.24292 & 0.436444 & $18285828+0026103$ & 17.8 & 15.5 & 14.0 & 13.4 & 13.2 & Yes \\
\hline 03-033 & 277.31750 & 0.306361 & $18291617+0018222$ & 21.0 & 18.0 & 14.3 & 11.8 & 10.1 & No \\
\hline 03-034 & 277.13470 & 0.493916 & $\ldots$ & 21.9 & 18.0 & $\ldots$ & $\ldots$ & $\ldots$ & No \\
\hline 03-035 & 277.26584 & 0.339027 & $18290394+0020212$ & 15.6 & 13.7 & 11.6 & 10.1 & 9.1 & No \\
\hline 03-037 & 277.43726 & 0.590944 & $18294502+0035264$ & 21.6 & 18.3 & 14.1 & 12.3 & 11.4 & No \\
\hline 03-038 & 277.12064 & 0.588888 & $18282871+0035206$ & 19.8 & 16.8 & 14.2 & 13.0 & 12.5 & No \\
\hline
\end{tabular}

Note. ${ }^{\text {a }}$ As in J. M. Brown et al., in preparation. 
Table 3

Observed Objects in Field AO6

\begin{tabular}{|c|c|c|c|c|c|c|c|c|c|}
\hline $\mathrm{ID}^{\mathrm{a}}$ & $\begin{array}{l}\text { R.A. } \\
\text { (deg) }\end{array}$ & $\begin{array}{l}\text { Decl. } \\
\text { (deg) }\end{array}$ & 2MASS Name & $\begin{array}{c}R \\
(\mathrm{mag})\end{array}$ & $\begin{array}{c}Z \\
(\mathrm{mag})\end{array}$ & $\begin{array}{c}J \\
(\mathrm{mag})\end{array}$ & $\begin{array}{c}H \\
(\mathrm{mag})\end{array}$ & $\begin{array}{c}K \\
(\mathrm{mag})\end{array}$ & Good S/N? \\
\hline 02-002 & 277.50937 & 0.940555 & $18300227+0056259$ & 13.5 & 12.3 & 10.8 & 10.1 & 9.9 & Yes \\
\hline 02-004 & 277.34213 & 0.672472 & $18292209+0040203$ & 23.0 & 17.4 & 13.3 & 11.1 & 10.0 & No \\
\hline $02-005$ & 277.43216 & 0.808805 & $18294372+0048308$ & 15.8 & 14.7 & 13.3 & 12.6 & 12.5 & Yes \\
\hline 02-006 & 277.22983 & 0.913722 & $18285512+0054496$ & 19.7 & 16.7 & 14.2 & 13.0 & 12.6 & No \\
\hline 02-007 & 277.18753 & 0.756916 & $18284499+0045239$ & 16.6 & 14.0 & 11.9 & 10.8 & 10.4 & Yes \\
\hline 02-008 & 277.51935 & 0.707138 & $18300459+0042247$ & 16.2 & 14.3 & 12.5 & 11.5 & 11.2 & Yes \\
\hline 02-009 & 277.31503 & 0.656249 & $18291564+0039227$ & 21.1 & 17.0 & 14.1 & 12.8 & 12.1 & No \\
\hline 02-010 & 277.54959 & 0.781416 & $18301185+0046519$ & 17.0 & 15.2 & 13.4 & 12.5 & 12.1 & No \\
\hline 02-013 & 277.47601 & 0.752222 & $18295424+0045074$ & 13.5 & 11.9 & 10.4 & 9.8 & 9.5 & Yes \\
\hline $02-014$ & 277.52570 & 0.709277 & $18300616+0042336$ & 14.2 & 12.3 & 9.8 & 8.6 & 7.5 & Yes \\
\hline 02-017 & 277.21396 & 0.873749 & $18285134+0052270$ & 16.1 & 13.5 & 11.9 & 11.3 & 11.0 & No \\
\hline 02-019 & 277.45401 & 0.731916 & $\cdots$ & $\cdots$ & $\ldots$ & $\ldots$ & $\ldots$ & $\ldots$ & No \\
\hline $02-021$ & 277.22470 & 0.765194 & $18285396+0045528$ & 18.7 & 15.7 & 13.3 & 12.1 & 11.5 & No \\
\hline $02-022$ & 277.35745 & 0.722611 & $\cdots$ & $\cdots$ & $\cdots$ & $\ldots$ & $\ldots$ & $\cdots$ & No \\
\hline $02-024$ & 277.35950 & 0.875499 & $\cdots$ & $\cdots$ & $\cdots$ & $\cdots$ & $\ldots$ & $\cdots$ & No \\
\hline $02-025$ & 277.56018 & 0.876055 & $18301440+0052352$ & 17.0 & 15.2 & 12.5 & 11.6 & 11.2 & Yes \\
\hline $02-027$ & 277.50992 & 0.869249 & $18300236+0052098$ & 17.6 & 15.1 & 13.1 & 12.0 & 11.6 & Yes \\
\hline $02-028$ & 277.45834 & 0.850444 & $18295003+0051014$ & 18.0 & 15.3 & 13.0 & 11.9 & 11.4 & Yes \\
\hline 02-029 & 277.42606 & 0.828666 & $18294221+0049431$ & 19.5 & 16.5 & 14.0 & 12.8 & 12.3 & Yes \\
\hline $02-030$ & 277.48447 & 0.657055 & $18295628+0039246$ & 17.0 & 15.2 & 13.1 & 12.1 & 11.7 & No \\
\hline 02-031 & 277.42199 & 0.817277 & $18294122+0049020$ & 19.3 & 16.2 & 13.8 & 12.5 & 11.9 & No \\
\hline $02-033$ & 277.28818 & 0.823861 & $\cdots$ & $\cdots$ & $\cdots$ & $\cdots$ & $\cdots$ & $\cdots$ & No \\
\hline $02-036$ & 277.25601 & 0.851166 & $\cdots$ & 21.2 & 18.8 & $\cdots$ & $\cdots$ & $\cdots$ & No \\
\hline 02-037 & 277.44699 & 0.665388 & $18294727+0039555$ & 17.0 & 15.2 & 13.6 & 12.6 & 12.1 & Yes \\
\hline $02-038$ & 277.46878 & 0.649944 & $\cdots$ & $\cdots$ & $\cdots$ & $\cdots$ & $\cdots$ & $\cdots$ & No \\
\hline 02-039 & 277.41428 & 0.715833 & $18293946+0042570$ & 18.6 & 16.6 & 13.6 & 12.4 & 11.9 & Yes \\
\hline 02-041 & 277.43964 & 0.934277 & $18294546+0056026$ & 19.6 & 16.4 & 13.5 & 12.1 & 11.4 & No \\
\hline 02-043 & 277.26514 & 0.753805 & $18290362+0045135$ & 19.9 & 16.4 & 13.5 & 12.1 & 11.6 & Yes \\
\hline 02-047 & 277.35171 & 0.805138 & $\cdots$ & $\ldots$ & $\cdots$ & $\cdots$ & $\ldots$ & $\ldots$ & No \\
\hline $02-048$ & 277.30933 & 0.787027 & $18291396+0047165$ & 20.8 & 17.8 & 15.1 & 13.8 & 13.4 & No \\
\hline 02-049 & 277.40204 & 0.775999 & $18293650+0046333$ & 19.5 & 16.2 & 13.5 & 12.2 & 11.7 & Yes \\
\hline $02-050$ & 277.28921 & 0.775749 & $18290943+0046343$ & 18.1 & 15.3 & 13.3 & 12.2 & 11.8 & No \\
\hline 02-051 & 277.55670 & 0.784777 & $18301365+0047055$ & 17.0 & 15.2 & 13.2 & 12.3 & 12.0 & Yes \\
\hline $02-052$ & 277.48480 & 0.835527 & $\cdots$ & $\cdots$ & $\cdots$ & $\cdots$ & $\cdots$ & $\cdots$ & No \\
\hline $02-053$ & 277.51263 & 0.879194 & $18300298+0052460$ & 19.8 & 16.8 & 14.5 & 13.7 & 13.2 & No \\
\hline $02-054$ & 277.35162 & 0.938861 & $18292426+0056189$ & 17.6 & 14.8 & 12.5 & 11.5 & 11.1 & No \\
\hline $02-055$ & 277.34671 & 0.859694 & $\cdots$ & $\cdots$ & $\cdots$ & $\cdots$ & $\cdots$ & $\cdots$ & No \\
\hline $02-056$ & 277.24307 & 0.934805 & $18285837+0056050$ & 20.4 & 16.6 & 14.1 & 13.1 & 12.6 & No \\
\hline 02-057 & 277.40082 & 0.704499 & $18293620+0042163$ & 18.8 & 16.6 & 13.3 & 11.9 & 11.3 & Yes \\
\hline $02-058$ & 277.30011 & 0.820249 & $\cdots$ & 22.7 & 20.4 & $\cdots$ & $\cdots$ & $\cdots$ & No \\
\hline 02-059 & 277.26154 & 0.767055 & $\cdots$ & $\cdots$ & $\cdots$ & $\cdots$ & $\cdots$ & $\cdots$ & No \\
\hline $02-060$ & 277.20172 & 0.798305 & $18284840+0047515$ & 17.2 & 15.1 & 13.2 & 12.2 & 11.9 & No \\
\hline 02-061 & 277.53561 & 0.811666 & $\cdots$ & 22.2 & 19.2 & $\cdots$ & $\cdots$ & $\cdots$ & No \\
\hline $02-062$ & 277.35211 & 0.922777 & $\cdots$ & $\ldots$ & $\cdots$ & $\cdots$ & $\cdots$ & $\cdots$ & No \\
\hline $02-063$ & 277.38480 & 0.900916 & $18293237+0054024$ & 18.0 & 15.6 & 13.5 & 12.5 & 12.1 & Yes \\
\hline $02-064$ & 277.45538 & 0.775388 & $\cdots$ & $\cdots$ & $\cdots$ & $\cdots$ & $\cdots$ & $\cdots$ & No \\
\hline $02-065$ & 277.47226 & 0.914527 & $18295337+0054523$ & 19.7 & 16.7 & 14.0 & 12.7 & 12.1 & No \\
\hline $02-068$ & 277.34466 & 0.815555 & $18292277+0048569$ & 19.5 & 16.6 & 14.0 & 12.8 & 12.4 & No \\
\hline 02-069 & 277.40829 & 0.888111 & $18293799+0053154$ & 22.7 & 18.9 & 15.6 & 14.3 & 13.7 & No \\
\hline 02-072 & 277.53726 & 0.789472 & $18300893+0047219$ & 17.7 & 15.4 & 13.5 & 12.4 & 12.0 & Yes \\
\hline $02-073$ & 277.27920 & 0.643722 & $18290698+0038378$ & 17.0 & 15.2 & 15.2 & 12.9 & 11.5 & No \\
\hline $02-074$ & 277.42184 & 0.962361 & $\cdots$ & 22.3 & 19.6 & $\cdots$ & $\cdots$ & $\cdots$ & No \\
\hline $02-076$ & 277.35800 & 0.744583 & $18292588+0044396$ & 20.4 & 16.2 & 13.7 & 12.5 & 12.2 & No \\
\hline 02-079 & 277.39325 & 0.951666 & $\cdots$ & $\cdots$ & $\cdots$ & $\cdots$ & $\cdots$ & $\cdots$ & No \\
\hline 02-080 & 277.44568 & 0.726527 & $18294682+0043396$ & 17.0 & 15.2 & 15.7 & 13.6 & 12.7 & No \\
\hline 02-081 & 277.39862 & 0.714861 & $18293575+0042499$ & 22.8 & 19.0 & 15.5 & 14.2 & 13.5 & No \\
\hline $02-083$ & 277.46017 & 0.728611 & $18295042+0043435$ & 17.8 & 15.4 & 13.0 & 11.5 & 10.5 & No \\
\hline 02-086 & 277.40628 & 0.748555 & $\cdots$ & $\cdots$ & $\cdots$ & $\cdots$ & $\cdots$ & $\cdots$ & No \\
\hline 02-088 & 277.38766 & 0.669555 & $18293301+0040087$ & 17.0 & 15.2 & 14.5 & 12.9 & 12.0 & No \\
\hline 02-093 & 277.44122 & 0.717305 & $\ldots$ & $\cdots$ & $\cdots$ & $\ldots$ & $\ldots$ & $\cdots$ & No \\
\hline
\end{tabular}

Note. ${ }^{\text {a }}$ As in J. M. Brown et al., in preparation. 


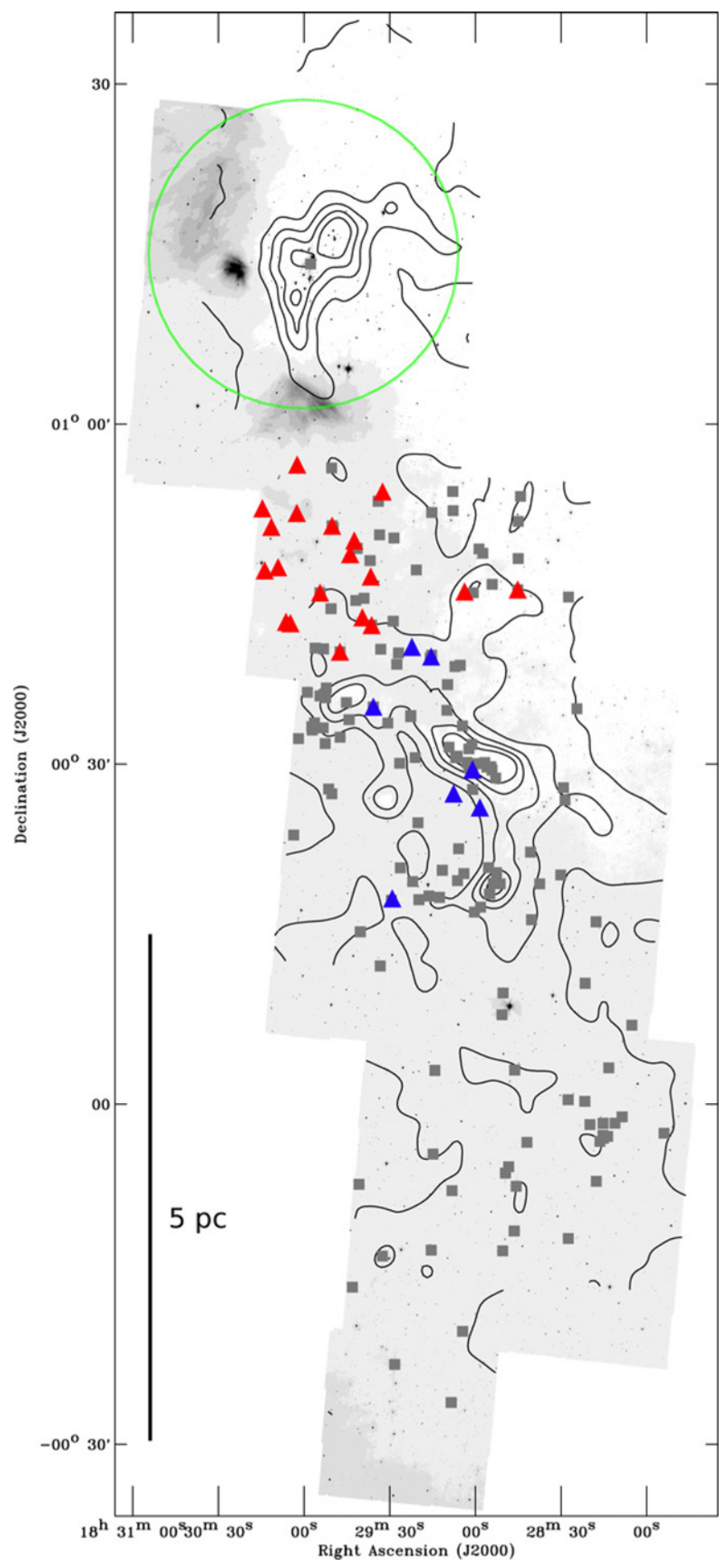

Figure 2. Observed objects overplotted on the $8.0 \mu \mathrm{m}$ IRAC 4 map of the Serpens Molecular Cloud. Young star candidates from this work are shown as red (field AO6) and blue (field AO5) triangles. Gray squares show the positions of the young stars with disks from Oliveira et al. (2010) for comparison. The contours $(5,10,15,20$, and $25 \mathrm{mag})$ of visual extinction are derived from the c2d extinction maps (Evans et al. 2007). The large green circle indicates the Serpens Core.

(A color version of this figure is available in the online journal.)

by Kenyon \& Hartmann (1995) is used, while for stars of later type, the relationship from Luhman et al. (2003) was adopted. The errors in $T_{\text {eff }}$ come directly from the errors in the spectral types (Section 3.1). The results can be found in Table 5.

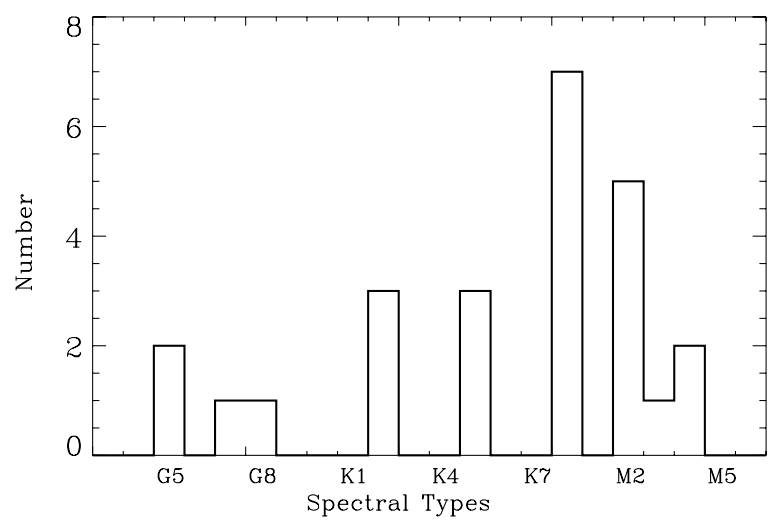

Figure 3. Distribution of spectral types of young diskless star candidates in Serpens based on the classification scheme described in Section 3.1.

\subsection{Stellar Luminosities}

Stellar luminosities are calculated by integrating the NEXTGEN model atmosphere (Hauschildt et al. 1999; Allard et al. 2000) corresponding to each object's spectral type, scaled to its dereddened optical and near-IR photometry and assuming a distance of $415 \pm 15$ pc (Dzib et al. 2010). Optical $R$ - and $Z$-band photometry for this region is available in the literature (Spezzi et al. 2010), although not all objects have been detected due to the high extinction toward some regions of the cloud. When optical photometry was not available, the Two Micron All Sky Survey (2MASS) photometry was used to scale the model atmospheres. Similar methods for luminosity estimates have been broadly used in the literature (Kenyon \& Hartmann 1995; van den Ancker et al. 1997; Alcalá et al. 2008; Merín et al. 2008; Oliveira et al. 2009, 2013; Mortier et al. 2011). The uncertainties in luminosity are derived from the errors in the distance and on the extinction ( $\pm 2 \mathrm{mag})$. The results can be found in Table 5.

Objects 03-009 and 03-027 have no available optical or nearIR photometry and therefore are left out of any further analysis. The following discussion concerns the remaining 23 objects.

\subsection{Spectral Energy Distributions}

The constructed SEDs for the 23 young diskless star candidates are shown in Figure 4. Besides the corresponding NEXTGEN model atmosphere (solid black line) and available optical/near-IR photometry from the literature, as discussed in the previous section, Spitzer mid-IR photometry is added where available. The IRAC (at 3.6, 4.5, 5.8, and $8.0 \mu \mathrm{m}$ ) and MIPS (at $24 \mu \mathrm{m}$ ) band fluxes were published by Harvey et al. (2006, 2007a, 2007b). In the figures, the crosses indicate the observed photometry, while the blue filled circles show the extinctioncorrected photometry. Individual extinction values were derived to best match the stellar photosphere, using the extinction law of Weingartner \& Draine (2001).

\subsection{Stellar Ages and Masses}

Once $T_{\text {eff }}$ and $L_{\text {star }}$ are known, it is possible to place the objects in an H-R diagram (Figure 5). With the aid of PMS evolutionary tracks, ages and masses can be derived for young stars by comparing each object's position to the isochrones and mass tracks of a particular model. Due to the physics and calibration of each model, the models of Baraffe et al. (2001) are used for stars less massive than $1.4 M_{\odot}$, while more massive stars are 
Table 4

Spectral Types of Objects with Classifiable Spectra

\begin{tabular}{|c|c|c|c|c|c|c|c|}
\hline Object ID & $\mathrm{SpT}$ & SpT Range & $\mathrm{H} \alpha^{\mathrm{a}}$ & $\mathrm{Li}^{\mathrm{a}}$ & IR Excess? & $\begin{array}{c}\text { X-Ray }{ }^{b, c} \\
\left(\text { counts s }^{-1}\right)\end{array}$ & $\begin{array}{l}\log L_{X}^{\mathrm{b}} \\
\left(\operatorname{erg~s}^{-1}\right)\end{array}$ \\
\hline 03-005 & M0 & M0.5 - K0 & Strong E & A & Yes & $0.0367(0.0303-0.0444)$ & 30.05 \\
\hline 03-009 & M0.5 & $\mathrm{M} 2-\mathrm{K} 7$ & Strong E & A & & $0.0193(0.0151-0.0243)$ & 29.62 \\
\hline 03-012 & M0 & $\mathrm{M} 2-\mathrm{K} 2$ & Weak E & A & & $0.0321(0.0244-0.0416)$ & 29.95 \\
\hline 03-017 & K5 & $\mathrm{K} 7-\mathrm{G} 7$ & A & $\cdots$ & & $0.0275(0.0207-0.0361)$ & 29.79 \\
\hline $03-026$ & M4 & M6 - M2 & Weak E & $\cdots$ & & $0.0048(0.0018-0.0086)$ & 29.09 \\
\hline $03-027$ & G8 & K5 - G0 & A & $\cdots$ & & $0.0075(0.0049-0.0109)$ & 29.47 \\
\hline $03-032$ & M4 & M6 - M2 & Weak E & $\cdots$ & & $0.0038(0.0007-0.0079)$ & 28.86 \\
\hline 02-002 & $\mathrm{K} 2$ & $\mathrm{~K} 7-\mathrm{K} 0$ & A & A & & $0.0908(0.0845-0.0976)$ & 30.35 \\
\hline $02-005$ & M0 & M0.5 - K5 & Weak E & $\cdots$ & & $0.0197(0.0175-0.0223)$ & 29.70 \\
\hline 02-007 & M2 & M3 - M0.5 & Weak E & A & Yes & $0.0569(0.0508-0.0639)$ & 30.10 \\
\hline 02-008 & $\mathrm{K} 2$ & $\mathrm{~K} 7-\mathrm{K} 0$ & $\cdots$ & A & & $0.0338(0.0299-0.0382)$ & 29.90 \\
\hline 02-013 & G5 & $\mathrm{G} 8-\mathrm{G} 2.5$ & A & A & & $0.0150(0.0127-0.0177)$ & 29.52 \\
\hline 02-014 & G7 & $\mathrm{K} 0-\mathrm{F} 0$ & Weak E & A & & $0.0184(0.0154-0.0219)$ & 29.60 \\
\hline $02-025$ & G5 & $\mathrm{G} 8-\mathrm{G} 2.5$ & A & $\cdots$ & & $0.0103(0.0084-0.0126)$ & 29.84 \\
\hline $02-027$ & M2 & M3 - M0.5 & Weak E & A & & $0.0112(0.0091-0.0139)$ & 29.41 \\
\hline $02-028$ & M3 & M4 - M0.5 & Weak E & A & Yes & $0.0073(0.0058-0.0092)$ & 29.29 \\
\hline 02-029 & M0 & M0.5 - K7 & Weak E & A & & $0.0065(0.0051-0.0081)$ & 29.20 \\
\hline $02-037$ & M2 & M3 - M0 & Strong E & A & Yes & $0.0073(0.0053-0.0098)$ & 29.27 \\
\hline 02-039 & K5 & $\mathrm{K} 7-\mathrm{K} 0$ & $\cdots$ & A & & $0.0079(0.0062-0.0100)$ & 29.32 \\
\hline $02-043$ & M0.5 & $\mathrm{M} 3-\mathrm{K} 7$ & Weak E & A & & $0.0079(0.0060-0.0102)$ & 29.24 \\
\hline 02-049 & M2 & M3 - M0.5 & Weak E & A & & $0.0042(0.0030-0.0056)$ & 28.92 \\
\hline $02-051$ & M0.5 & $\mathrm{M} 2-\mathrm{K} 7$ & Weak E & A & & $0.0069(0.0049-0.0094)$ & 29.27 \\
\hline $02-057$ & K5 & $\mathrm{K} 7-\mathrm{K} 0$ & A & A & Yes & $0.0062(0.0046-0.0082)$ & 29.13 \\
\hline $02-063$ & M0.5 & M3 - M0 & Weak E & A & & $0.0032(0.0020-0.0048)$ & 28.92 \\
\hline $02-072$ & M2 & M3 - M0.5 & Strong E & A & Yes & $0.0051(0.0034-0.0073)$ & 29.11 \\
\hline
\end{tabular}

Notes.

${ }^{\text {a }}$ E denotes emission and A denotes absorption.

${ }^{\mathrm{b}}$ From J. M. Brown et al., in preparation.

${ }^{\mathrm{c}}$ In parenthesis is the $95 \%$ confidence range.

compared to the models of Siess et al. (2000). The results are displayed in Table 5.

Due to the relatively old ages $\left(>2 \times 10^{7} \mathrm{yr}\right.$ ) derived for objects 02-005, 02-008, 02-025, and 02-039, it is unclear whether they indeed belong to the cloud, given the uncertainty of PMS evolutionary models. It is worth noting that the spectra of objects 02-005 and 02-025 do not show Li I, while the line is seen in the spectra of 02-008 and 02-039. At this point it is difficult to confirm membership and these four objects are left out of the further discussion. The remaining 19 objects are confirmed new members of Serpens.

\subsubsection{Infrared Excess}

It can be seen from Figure 4 that 6 out of 19 new confirmed members of Serpens (03-005, 02-007, 02-028, 02-037, 02-057, and 02-072) show some IR excess. Objects 02-007 and 02-028 show excess emission throughout the Spitzer bands. This is considered weak IR excess as it is lower than the median excess for objects in Taurus (Furlan et al. 2006), the prototype for full disks. The other four objects have SEDs indicative of cold or transitional disks, where no excess is seen in the near- to midIR, and a more substantial excess is present at mid- to far-IR. Object 03-005 was indeed previously confirmed as a cold disk with Spitzer/IRS spectra (Merín et al. 2010). Objects 02-037, 02-057, and 02-072 are new cold disk candidates. None of the four older objects, not yet confirmed to be members of the cloud, show any IR excess.

Following White \& Basri (2003), we identify $\mathrm{H} \alpha$ emission in Table 4 as strong (full width at $10 \%$ of peak intensity higher than $\left.270 \mathrm{~km} \mathrm{~s}^{-1}\right)$ or weak $\left(\mathrm{H} \alpha 10 \%<270 \mathrm{~km} \mathrm{~s}^{-1}\right)$. In addition, in Table 4 " $\mathrm{A}$ " indicates that $\mathrm{H} \alpha$ is seen in absorption. Three out of the 19 new members show strong emission of the $\mathrm{H} \alpha$ line. These objects (03-005, 02-037, and 02-072) also show IR excess, indicating that disks are still present. None of the confirmed diskless sources show strong $\mathrm{H} \alpha$ emission, albeit some do show weak $\mathrm{H} \alpha$ emission, which can be attributed to chromospheric activity (White \& Basri 2003).

Out of 86 stars observed by FLAMES, only 25 objects are bright enough for spectral classification. Out of these 25 objects analyzed, 19 are confirmed new members of Serpens, while the other 6 could not be confirmed or rejected at this point. Out of the new confirmed members of the cloud, two-thirds are shown to be diskless, while the other one-third show IR excess weaker than the median of Taurus.

\section{DISCUSSION}

The most straightforward explanation for why the disks around these newly confirmed young stars have already dissipated would be that these stars are systematically a few million years older than the stars with disks in the same area of Serpens (Oliveira et al. 2013). The higher ages would be sufficient for their disks to have evolved and dissipated. This could be the case for the older objects 02-005, 02-008, 02-025, and 02-039, if these objects indeed belong to the cloud. Another possibility would be that these diskless stars are consistently more massive than the disk-bearing stars in the same area of Serpens of the same age. It has been shown that disk lifetimes are related to the host star mass, with higher mass stars having disks that 
Table 5

Stellar Parameters for the Young Diskless Stars in Serpens

\begin{tabular}{|c|c|c|c|c|c|}
\hline Object ID & $\begin{array}{c}A v \\
(\mathrm{mag})\end{array}$ & $\begin{array}{l}T_{\text {eff }} \\
(\mathrm{K})\end{array}$ & $\begin{array}{c}L_{*} \\
\left(L_{\odot}\right)\end{array}$ & $\begin{array}{l}\text { Mass } \\
\left(M_{\odot}\right)\end{array}$ & $\begin{array}{l}\text { Age } \\
\text { (Myr) }\end{array}$ \\
\hline 03-005 & 4.6 & $3850_{-65}^{+1400}$ & $1.52_{-0.68}^{+1.22}$ & $1.06_{-0.08}^{+0.18}$ & $1.01_{-1.01}^{+20.31}$ \\
\hline 03-012 & 3.2 & $3850_{-290}^{+1050}$ & $0.73_{-0.33}^{+0.58}$ & $0.98_{-0.33}^{+0.06}$ & $3.06_{-1.94}^{+27.13}$ \\
\hline 03-017 & 13.6 & $4350_{-290}^{+1280}$ & $5.87_{-2.64}^{+4.72}$ & $1.60_{-1.60}^{+0.12}$ & $1.00_{-1.00}^{+6.74}$ \\
\hline 03-026 & 0.0 & $3270_{-280}^{+290}$ & $0.13_{-0.06}^{+0.11}$ & $0.26_{-0.15}^{+0.25}$ & $2.67_{-2.67}^{+7.06}$ \\
\hline 03-032 & 0.0 & $3270_{-280}^{+290}$ & $0.06_{-0.03}^{+0.05}$ & $0.23_{-0.14}^{+0.27}$ & $6.83_{-5.73}^{+23.38}$ \\
\hline $02-002$ & 2.0 & $4900_{-840}^{+350}$ & $3.01_{-1.35}^{+2.42}$ & $1.79_{-1.10}^{+0.31}$ & $3.95_{-3.95}^{+3.79}$ \\
\hline $02-005$ & 0.0 & $3850_{-65}^{+500}$ & $0.13_{-0.06}^{+0.11}$ & $0.73_{-0.13}^{+0.09}$ & $30.71_{-27.23}^{+47.73}$ \\
\hline $02-007$ & 3.4 & $3560_{-145}^{+225}$ & $1.14_{-0.51}^{+0.91}$ & $0.75_{-0.13}^{+0.22}$ & $0.41_{-0.41}^{+1.03}$ \\
\hline 02-008 & 4.0 & $4900_{-840}^{+350}$ & $1.09_{-0.49}^{+0.87}$ & $1.18_{-1.18}^{+0.00}$ & $20.16_{-17.29}^{+22.94}$ \\
\hline 02-013 & 3.2 & $5770_{-250}^{+75}$ & $8.22_{-3.69}^{+6.60}$ & $1.84_{-0.35}^{+0.40}$ & $6.69_{-2.55}^{+5.10}$ \\
\hline $02-014$ & 10.3 & $5630_{-380}^{+1570}$ & $86.46_{-38.86}^{+69.45}$ & $3.35_{-0.09}^{+0.09}$ & $1.71_{-0.44}^{+0.44}$ \\
\hline $02-025$ & 5.1 & $5770_{-250}^{+75}$ & $1.92_{-0.86}^{+1.54}$ & $1.35_{-1.35}^{+0.01}$ & $24.50_{-15.24}^{+980.15}$ \\
\hline $02-027$ & 2.8 & $3560_{-145}^{+225}$ & $0.34_{-0.15}^{+0.27}$ & $0.55_{-0.13}^{+0.26}$ & $2.93_{-1.42}^{+4.51}$ \\
\hline $02-028$ & 3.5 & $3415_{-145}^{+370}$ & $0.40_{-0.18}^{+0.32}$ & $0.45_{-0.14}^{+0.38}$ & $1.44_{-0.99}^{+4.56}$ \\
\hline $02-029$ & 4.6 & $3850_{-65}^{+210}$ & $0.23_{-0.11}^{+0.19}$ & $0.81_{-0.09}^{+0.09}$ & $15.32_{-8.32}^{+16.65}$ \\
\hline $02-037$ & 2.8 & $3560_{-145}^{+290}$ & $0.21_{-0.10}^{+0.17}$ & $0.53_{-0.14}^{+0.27}$ & $5.68_{-3.10}^{+11.80}$ \\
\hline 02-039 & 5.1 & $4350_{-290}^{+900}$ & $0.49_{-0.22}^{+0.39}$ & $1.03_{-0.18}^{+0.25}$ & $19.55_{-11.23}^{+638.39}$ \\
\hline $02-043$ & 5.4 & $3785_{-370}^{+275}$ & $0.47_{-0.21}^{+0.38}$ & $0.85_{-0.37}^{+0.20}$ & $4.71_{-3.58}^{+4.98}$ \\
\hline 02-049 & 4.8 & $3560_{-145}^{+225}$ & $0.39_{-0.17}^{+0.31}$ & $0.57_{-0.12}^{+0.26}$ & $2.59_{-1.38}^{+3.67}$ \\
\hline 02-051 & 1.9 & $3785_{-225}^{+275}$ & $0.25_{-0.11}^{+0.20}$ & $0.78_{-0.25}^{+0.07}$ & $10.36_{-5.69}^{+15.54}$ \\
\hline $02-057$ & 6.6 & $4350_{-290}^{+900}$ & $0.96_{-0.43}^{+0.77}$ & $1.31_{-1.31}^{+\dagger}$ & $7.56_{-4.61}^{+35.04}$ \\
\hline $02-063$ & 3.0 & $3785_{-370}^{+65}$ & $0.24_{-0.11}^{+0.19}$ & $0.78_{-0.39}^{+0.06}$ & $10.67_{-8.16}^{+15.66}$ \\
\hline 02-072 & 3.0 & $3650_{-235}^{+135}$ & $0.24_{-0.11}^{+0.19}$ & $0.63_{-0.23}^{+0.15}$ & $7.21_{-4.65}^{+9.11}$ \\
\hline
\end{tabular}

dissipated faster (Carpenter et al. 2006; Kennedy \& Kenyon 2009).

Figure 6 shows the mass and age distributions for our sample of newly confirmed members of Serpens (solid red lines), and for the disk-bearing young stars in the same region (dot-dashed black lines; Oliveira et al. 2013). It is immediately clear that, although not perfect copies of each other (KS statistical tests show that the hypothesis that they are drawn from different distributions is not supported, with probabilities $P=24 \%$ and $10 \%$ for the distributions of ages and masses, respectively), the distributions of both stellar ages and masses of diskless young stars have the same spread, and even the same peak locations, as those of disk-bearing stars in the same region. Once again it is important to note that only the confirmed new members are taken into consideration here. If the four older stars (Section 3.5) are indeed members of the cloud, the statistical significance would change for the age distribution $(P=0.09$ and 0.4 for the distributions of ages and masses, respectively). However, it is true that the bulk of this new population is not consistently shifted toward higher ages.

Although we acknowledge that the sample studied here does not necessarily represent the entire population of diskless young stars in Serpens, the similarity of the distributions suggests that ages and stellar masses are not absolute drivers of disk evolution. Other parameters must play an important role, such that stars of similar age and mass end up having such different disks (or lack thereof). That is not to say that either parameter is not important for disk evolution; the big picture remains that disks evolve with time and, statistically, older stars have more evolved (or no) disks compared to young stars. However, a complete theory of protoplanetary disk evolution must take into account that stars of similar age in the same environment do present large variety of disk structures (e.g., Furlan et al. 2006; Fang et al. 2009; Oliveira et al. 2010, 2013; Sicilia-Aguilar et al. 2011) or no disks at all.

Similar results were seen by Feigelson \& Montmerle (1999). From a reliable X-ray sample from the ROSAT satellite, stars were characterized spectroscopically and placed in H-R diagrams. They report that in the Chamaeleon I and Taurus-Auriga star-forming regions, no significant differences are seen in the age distributions of disk-bearing (class II) and diskless (class III) stars. In that work, the authors discuss two immediate consequences of these results: (1) an underestimate of cloud star formation rates for samples dominated by class II stars and (2) the high number of class III stars in young regions implies that many (at least one-half of low-mass stars in Chamaeleon I) lose their disks within 1 Myr. In that work, the authors already argued that there is no preferred disk lifetime, since disk-bearing and diskless stars coexist along the Hayashi track.

More recently, aided by Chandra observations and optical spectroscopy, Winston et al. (2009, 2010) showed very similar age distributions for class II and class III stars in the Serpens Core and NGC 1333. They argued that young stellar populations with and without disks show indistinguishable spatial and age distributions, suggesting that class III stars are not typically older stars, but rather stars that lost their disks quicker.

In addition, Cieza et al. (2007) and Beccari et al. (2010) have studied the differences between classical $\mathrm{T}$ Tauri stars (CTTS; young stars actively accreting material from their disks) and weak-line T Tauri stars (WTTS; young stars that show no signs of accretion). Correspondingly, both studies find no significant difference in the age distributions of CTTS and WTTS. However, it is important to note that not all WTTS are bona-fide class III objects. Many WTTS have disks (even thick disks) but for some reason are no longer accreting material onto their host stars. Therefore, what these results show is that there is no significant difference in the age distribution of stars that are actively accreting from their disks and stars that are not.

Several different processes actively being studied could possibly be responsible for the fast dissipation of some disks, while others last many millions of years. For instance, a relationship between the disk mass (from $1.3 \mathrm{~mm}$ observations with the Submillimeter Array) and stellar mass has been shown to be real for a complete disk sample in Taurus by Andrews et al. (2013). If confirmed for other complete and unbiased samples, this result puts strong constraints on theories for disk evolution and planet formation. The stellar mass would determine the amount of material available in disks from which planets can form.

Another interesting process that could be decisive on disk evolution timescales is photoevaporation. Theoretical calculations predict that energetic UV and X-ray photons emitted by the central star could heat the surface of the disk and cause pressuredriven hydrodynamic mass outflows from the disk (Hollenbach et al. 2000; Clarke et al. 2001; Alexander et al. 2006a, 2006b; Ercolano et al. 2009; Gorti et al. 2009; Owen et al. 2010). Coupled with the disk viscous evolution, disks could be dispersed in $\sim 10^{6}$ Myr. Observational confirmation of photoevaporation is an active field that has already produced some interesting results (e.g., Pascucci \& Sterzik 2009; Pascucci et al. 2012).

Just as exciting, and perhaps even more observationally challenging, is the effect of multiplicity on disk evolution. Since the majority of low-mass stars form in multiple systems, binary 

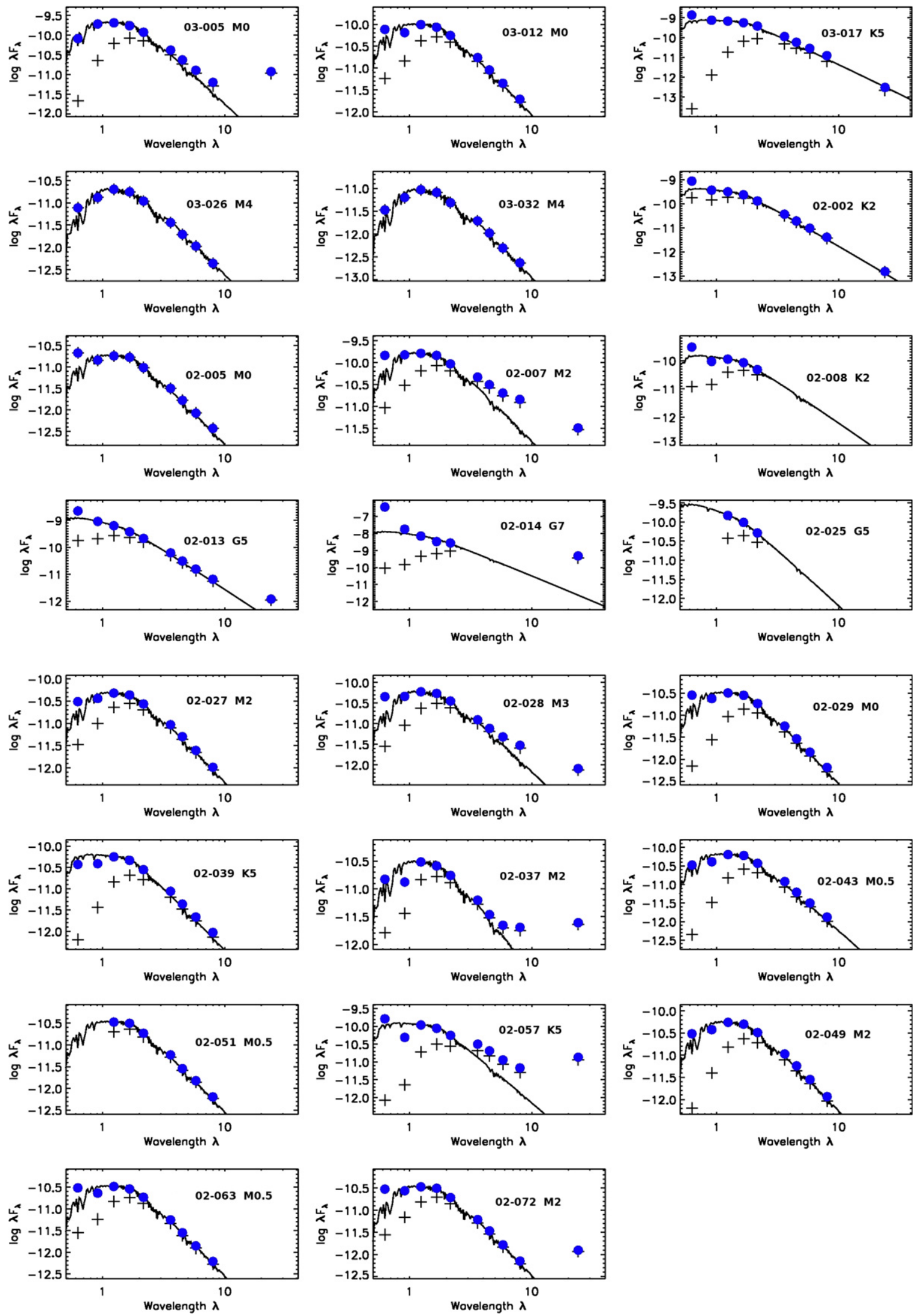

Figure 4. Spectral energy distributions of the new young diskless population in Serpens. Each SED has the corresponding object ID (as in Tables 2 and 3). The solid black line indicates the NEXTGEN atmosphere model for the corresponding spectral type of each object, also shown in the plot. Plus signs indicate the observed photometry, while solid blue circles denote the dereddened photometry. No SEDs are shown for the objects without photometric data (objects 03-009 and 03-027).

(A color version of this figure is available in the online journal.) 

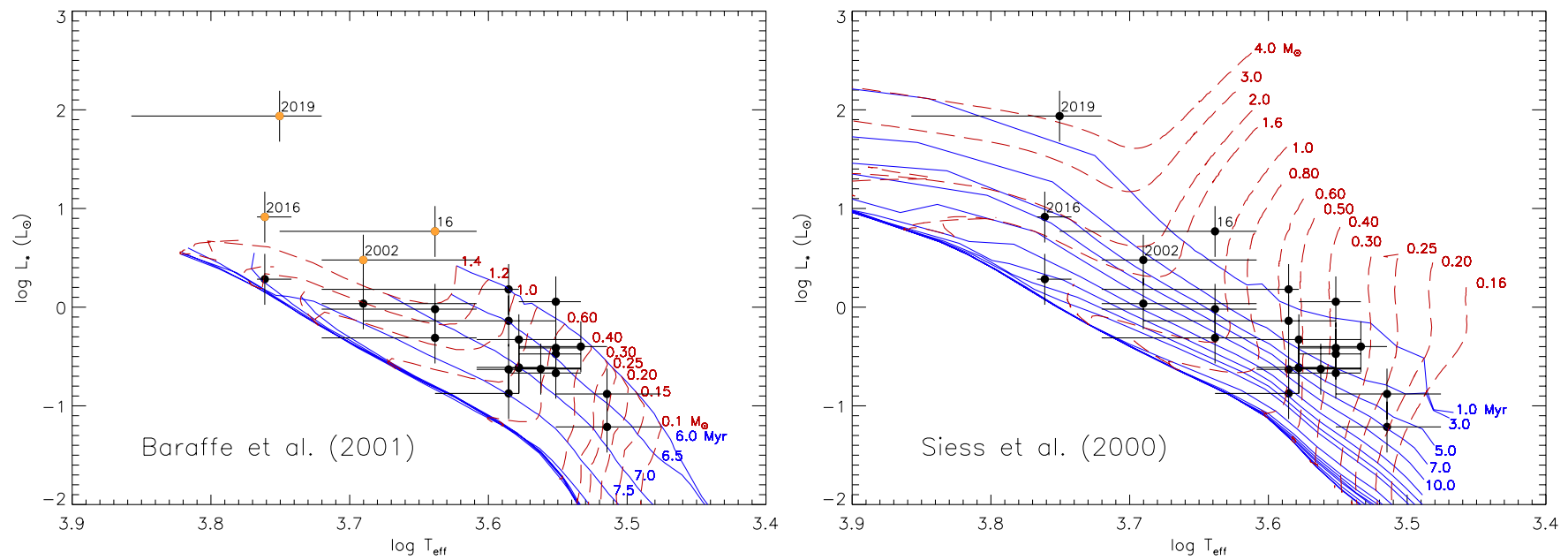

Figure 5. H-R diagrams overlaid with the pre-main-sequence evolutionary models of Baraffe et al. (2001), left, and Siess et al. (2000), right. (A color version of this figure is available in the online journal.)

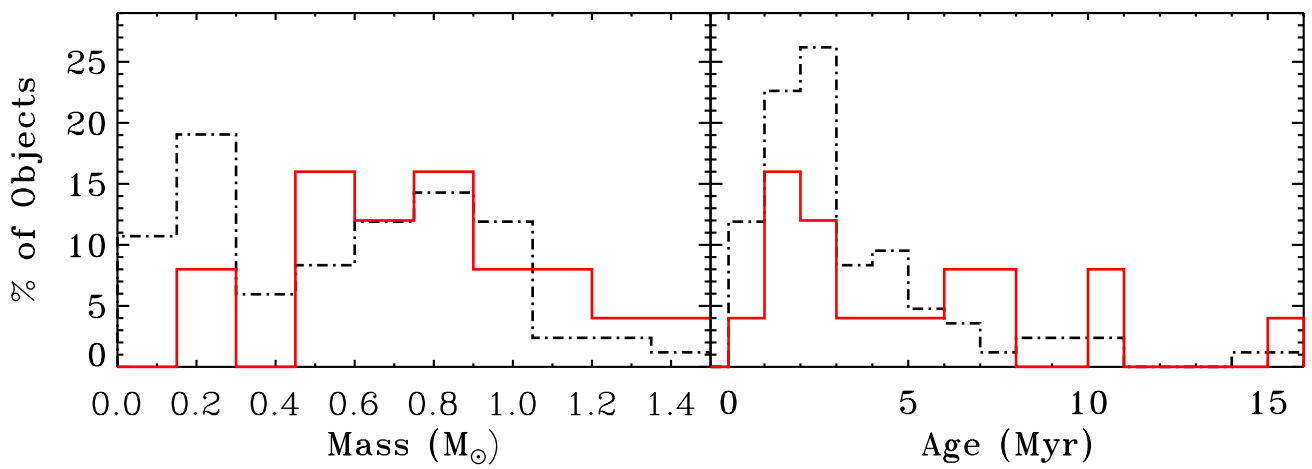

Figure 6. Distribution of masses and ages of the new young objects confirmed in Serpens (solid red lines). The distribution of the previously known young stellar objects in Serpens (Oliveira et al. 2013) is shown for comparison (dot-dashed black lines).

(A color version of this figure is available in the online journal.)

companions can affect disk evolution directly. Kraus et al. (2012) showed these effects for the first time on a complete sample in Taurus-Auriga. They found that tidal influence of a close binary ( $\lesssim 40$ AU) significantly accelerates disk dispersal, with disks being completely dissipated within 1 Myr for $\sim$ twothirds of close binaries. The same is true for only a small fraction of wide binaries and single stars $(\sim 10 \%-20 \%)$ whose disks are dispersed within 2-3 Myr. These exciting results must be confirmed with similar observations of complete samples of young stars in different star-forming regions, of different mean ages and environments.

\section{CONCLUSIONS}

We have confirmed, for the first time, a significant population of young diskless stars discovered at X-ray wavelengths that are members of the Serpens Molecular Cloud.

We have determined spectral types, effective temperatures, and luminosities using optical spectroscopy from VLT/FLAMES and optical/near-IR photometry.

Stellar ages and masses for 23 stars were derived based on a comparison with PMS evolutionary tracks. While 19 young stars were confirmed to be members of Serpens, the remaining four could not be conclusively shown to have ages consistent with cluster membership. Six of these 19 confirmed stars show weak IR excess (below the Taurus median), while the other 13 are truly diskless sources.
We find that the age and mass distributions of this new diskless stellar population are remarkably similar to those of the diskbearing young stars in the same region, studied previously by Oliveira et al. (2013).

This similarity may hold significant implications for the picture of disk evolution in which the main factors that determine the disk fraction are mass and age. If confirmed for the larger sample of young diskless star candidates, our results indicate that other, yet poorly understood observationally, characteristics may play an important role as well.

This work is based on observations made with the ESO Telescopes at the Paranal Observatories under program ID 083.C-0766(B). The authors would like to thank the anonymous referee for her/his suggestions, which greatly improved this manuscript.

\section{REFERENCES}

Alcalá, J. M., Spezzi, L., Chapman, N., et al. 2008, ApJ, 676, 427

Alexander, R. D., Clarke, C. J., \& Pringle, J. E. 2006a, MNRAS, 369, 216 Alexander, R. D., Clarke, C. J., \& Pringle, J. E. 2006b, MNRAS, 369, 229

Allard, F., Hauschildt, P. H., \& Schweitzer, A. 2000, ApJ, 539, 366

Andrews, S. M., Rosenfeld, K. A., Kraus, A. L., \& Wilner, D. J. 2013, ApJ, 771,129

Baraffe, I., Chabrier, G., Allard, F., \& Hauschildt, P. 2001, in ASP Conf. Proc. 243, From Darkness to Light: Origin and Evolution of Young Stellar Clusters, ed. T. Montmerle \& P. André (San Francisco, CA: ASP), 571

Beccari, G., Spezzi, L., De Marchi, G., et al. 2010, ApJ, 720, 1108 
Carpenter, J. M., Mamajek, E. E., Hillenbrand, L. A., \& Meyer, M. R. 2006, ApJL, 651, L49

Cieza, L., Padgett, D. L., Stapelfeldt, K. R., et al. 2007, ApJ, 667, 308

Clarke, C. J., Gendrin, A., \& Sotomayor, M. 2001, MNRAS, 328, 485

Dzib, S., Loinard, L., Mioduszewski, A. J., et al. 2010, ApJ, 718, 610

Ercolano, B., Clarke, C. J., \& Drake, J. J. 2009, ApJ, 699, 1639

Evans, N. J., II, et al. 2007, Delivery Documentation, http://ssc.spitzer.caltech. edu/legacy/c2dhistory.html

Fang, M., van Boekel, R., Wang, W., et al. 2009, A\&A, 504, 461

Feigelson, E. D., \& Kriss, G. A. 1981, ApJL, 248, L35

Feigelson, E. D., \& Montmerle, T. 1999, ARA\&A, 37, 363

Feigelson, E., Townsley, L., Güdel, M., \& Stassun, K. 2007, in Protostars and Planets V, ed. B. Reipurth, D. Jewitt, \& K. Keil (Tucson, AZ: Univ. Arizona Press), 313

Furlan, E., Hartmann, L., Calvet, N., et al. 2006, ApJS, 165, 568

Getman, K. V., Feigelson, E. D., Grosso, N., et al. 2005, ApJS, 160, 353

Gorti, U., Dullemond, C. P., \& Hollenbach, D. 2009, ApJ, 705, 1237

Güdel, M., Briggs, K. R., Arzner, K., et al. 2007, A\&A, 468, 353

Haisch, K. E., Jr., Lada, E. A., \& Lada, C. J. 2001, ApJL, 553, L153

Hartmann, L. 2003, ApJ, 585, 398

Harvey, P. M., Chapman, N., Lai, S.-P., et al. 2006, ApJ, 644, 307

Harvey, P., Merín, B., Huard, T. L., et al. 2007a, ApJ, 663, 1149

Harvey, P. M., Rebull, L. M., Brooke, T., et al. 2007b, ApJ, 663, 1139

Hauschildt, P. H., Allard, F., Ferguson, J., Baron, E., \& Alexander, D. R. 1999, ApJ, 525, 871

Hernández, J., Hartmann, L., Calvet, N., et al. 2008, ApJ, 686, 1195

Hollenbach, D. J., Yorke, H. W., \& Johnstone, D. 2000, in Protostars and Planets IV, ed. V. Mannings, A. P. Boss, \& S. S. Russell (Tucson, AZ: Univ. Arizona Press), 401

Kennedy, G. M., \& Kenyon, S. J. 2009, ApJ, 695, 1210

Kenyon, S. J., \& Hartmann, L. 1995, ApJS, 101, 117

Kraus, A. L., Ireland, M. J., Hillenbrand, L. A., \& Martinache, F. 2012, ApJ, 745,19

Luhman, K. L., Stauffer, J. R., Muench, A. A., et al. 2003, ApJ, 593, 1093

Merín, B., Brown, J. M., Oliveira, I., et al. 2010, ApJ, 718, 1200

Merín, B., Jørgensen, J., Spezzi, L., et al. 2008, ApJS, 177, 551
Montes, D., Martin, E. L., Fernandez-Figueroa, M. J., Cornide, M., \& de Castro, E. 1997, A\&AS, 123, 473

Mora, A., Merín, B., Solano, E., et al. 2001, A\&A, 378, 116

Mortier, A., Oliveira, I., \& van Dishoeck, E. F. 2011, MNRAS, 418, 1194

Muzerolle, J., Hillenbrand, L., Calvet, N., Briceño, C., \& Hartmann, L. 2003, ApJ, 592, 266

Natta, A., Testi, L., Muzerolle, J., et al. 2004, A\&A, 424, 603

Oliveira, I., Merín, B., Pontoppidan, K. M., \& van Dishoeck, E. F. 2013, ApJ, 762,128

Oliveira, I., Merín, B., Pontoppidan, K. M., et al. 2009, ApJ, 691, 672

Oliveira, I., Pontoppidan, K. M., Merín, B., et al. 2010, ApJ, 714, 778

Owen, J. E., Ercolano, B., Clarke, C. J., \& Alexander, R. D. 2010, MNRAS, 401, 1415

Padgett, D. L., Cieza, L., Stapelfeldt, K. R., et al. 2006, ApJ, 645, 1283

Pascucci, I., Gorti, U., \& Hollenbach, D. 2012, ApJL, 751, L42

Pascucci, I., \& Sterzik, M. 2009, ApJ, 702, 724

Preibisch, T., Kim, Y.-C., Favata, F., et al. 2005, ApJS, 160, 401

Randich, S., Pallavicini, R., Meola, G., Stauffer, J. R., \& Balachandran, S. C. 2001, A\&A, 372, 862

Scelsi, L., Sacco, G., Affer, L., et al. 2008, A\&A, 490, 601

Sicilia-Aguilar, A., Henning, T., Dullemond, C. P., et al. 2011, ApJ, 742, 39

Siess, L., Dufour, E., \& Forestini, M. 2000, A\&A, 358, 593

Spezzi, L., Merín, B., Oliveira, I., van Dishoeck, E. F., \& Brown, J. M. 2010, A\&A, 513, A38

Telleschi, A., Güdel, M., Briggs, K. R., Audard, M., \& Scelsi, L. 2007, A\&A, 468,443

van den Ancker, M. E., The, P. S., Feinstein, A., et al. 1997, A\&AS, 123,63

Walter, F. M., Brown, A., Mathieu, R. D., Myers, P. C., \& Vrba, F. J. 1988, AJ, 96, 297

Walter, F. M., \& Kuhi, L. V. 1981, ApJ, 250, 254

Weingartner, J. C., \& Draine, B. T. 2001, ApJ, 548, 296

White, R. J., \& Basri, G. 2003, ApJ, 582, 1109

Winston, E., Megeath, S. T., Wolk, S. J., et al. 2007, ApJ, 669, 493

Winston, E., Megeath, S. T., Wolk, S. J., et al. 2009, AJ, 137, 4777

Winston, E., Megeath, S. T., Wolk, S. J., et al. 2010, AJ, 140, 266 